This is the author's final, peer-reviewed manuscript as accepted for publication. The publisher-formatted version may be available through the publisher's web site or your institution's library.

\title{
Dynamic optimization of batch diafiltration processes
}

M. Fikar, Z. Kovács, P. Czermak

\section{How to cite this manuscript (APA format)}

If you make reference to this version of the manuscript, use the following citation format:

Fikar, M., Kovács, Z., Czermak, P. (2010). Dynamic optimization of batch diafiltration processes. Retrieved from http://krex.ksu.edu

\section{Published Version Information}

Citation: Fikar, M., Kovács, Z., Czermak, P. (2010). Dynamic optimization of batch diafiltration processes. Journal of Membrane Science, 355, 168-174.

Copyright: (c) 2010 Elsevier B.V. All rights reserved.

Digital Object Identifier (DOI): 10.1016/j.memsci.2010.03.019

Publisher's Link: http://www.sciencedirect.com/science/journal/03767388

This item was retrieved from the K-State Research Exchange (K-REx), the institutional repository of Kansas State University. K-REx is available at http://krex.ksu.edu 
1

2

3

\title{
Dynamic optimization of batch diafiltration processes
}

\author{
M. Fikar ${ }^{\mathrm{a}, *}$, Z. Kovács ${ }^{\mathrm{b}, * *}$, P. Czermak ${ }^{\mathrm{b}, \mathrm{c}}$ \\ ${ }^{a}$ Department of Information Engineering and Process Control FCFT, Slovak University \\ of Technology, Bratislava, Slovakia \\ ${ }^{b}$ Institute of Biopharmaceutical Technology, University of Applied Sciences \\ Giessen-Friedberg, Giessen, Germany \\ ${ }^{c}$ Department of Chemical Engineering, Kansas State University, Manhattan, Kansas, \\ USA
}

\begin{abstract}
A comprehensive modeling approach is proposed for the dynamic simulation and operation optimization of batch diafiltration processes. We provide a unified technology for water utilization control that addresses generality versus special cases. A rigorous dynamical model of the diafiltration process with concentration-dependent rejections of solutes is developed. We determine the optimal time-dependent profile of the diluant flow for the entire process using dynamic optimization methods. The results show that optimal process operation needs not to be any of the conventional diafiltration concepts. The presented optimization technique is a useful tool for improving the performance of a membrane diafiltration process.
\end{abstract}

Keywords: membrane filtration, diafiltration, mathematical modeling, dynamic optimization

\section{Introduction}

Membrane filtration processes are usually designed to fulfill dual objectives: (1) to separate certain solutes from the process liquor and (2) to concentrate the purified solution in order to obtain a final product. We examine a batch diafiltration process that meets these simultaneous objectives.

\footnotetext{
${ }^{*}$ Corresponding author

** Principal corresponding author

Email addresses: miroslav.fikar@stuba.sk (M. Fikar), kovacs.zoltan@tg.fh-giessen.de (Z. Kovács) 
There is no exact and uniform definition for the term diafiltration. Indeed, the terminology currently being used is conflicting. In this paper, we use the term diafiltration in its broad sense referring to the actual technological goal. Thus, diafiltration is a membrane-assisted process that is designed to achieve the twin-objectives of concentrating and purifying a multi-solute system according to a specific wash-water utilization strategy. In this context, batch diafiltration is a complex process that may involve a sequence of consecutive operational steps.

\section{Basic operational modes}

We consider three frequently used operational modes. These are the concentration mode $(C)$, the constant-volume dilution mode (CVD), and the variable-volume dilution mode (VVD). They differ from each other in the utilization of wash-water.

In concentration mode, no wash-water is introduced into the feed tank, thus resulting in a continuous volume decrease of the feed.

In CVD, the feed volume is kept constant by continuously adding a diluant at a rate equal to the permeation rate. We point out, that although we use the term diafiltration in a wider context, very often this single operation mode (e.g. CVD) is identified as diafiltration in the literature.

The VVD is an operation mode in which fresh water is continuously added to the feed tank at a rate that is proportional but less than the permeate flow. This causes a simultaneous concentration of macrosolute, and removal of microsolute. This operation has been proposed by Jaffrin and Charrier (Jaffrin and Charrier, 1994), analyzed in some detail by Tekić et al. and Krstić et al. (Tekić et al., 2002; Krstić et al., 2004), and recently revised by Foley (Foley, 2006a).

\section{Conventional diafiltration techniques}

According to our terminological definition, an operation mode does operate with fixed operational settings. A diafiltration process, in contrast, is usually constructed by changing the settings of wash-water addition (i.e. switching to another operational mode) according to a pre-defined schedule. The three most commonly used concepts of diafiltration are as follows:

- Traditional diafiltration (TD) process involves three consecutive steps (i.e. operational modes). First, a pre-concentration is used to reduce 
the fluid volume and remove some of the microsolute. Then, a constantvolume dilution step is employed to "wash out" the microsolute by adding a washing solution (e.g. diluant) into the system at a rate equal to the permeate flow rate. Thus, the volume of the solution in the feed tank is kept constant during this operational mode. Finally, a postconcentration is used to obtain the final volume and concentrate the macrosolute to the final concentration due to the specific technological demands.

- Pre-concentration combined with variable-volume dilution (PVVD): This concept is credited to Foley (Foley, 2006b). It is a two step process in which the solution is first pre-concentrated to an intermediate macrosolute concentration and then subjected to VVD to reach the final desired concentrations of both solutes.

- Intermittent feed diafiltration (IFD) is an operation mode in which the diluant is added intermittent (Wang et al., 2008). IFD starts with a pre-concentration step. Then, a washing solution is added into the feed tank to set back the initial feed volume. These two steps are repeated several times. Finally, a post-concentration step is applied to achieve the final volume.

Another diafiltration approach has recently been introduced by Takači et al. (Takači et al., 2009). Instead of a stepwise water utilization strategy, the authors have considered the ratio of diluant flow to permeate flow as a continuous function of the operational time. Some linear, logarithmic, and exponential functions have been studied, and their impact on the required diafiltration time was simulated.

Several studies have examined the different types of diafiltration techniques in terms of process time and wash-water requirement (Jaffrin and Charrier, 1994; Tekić et al., 2002; Krstić et al., 2004; Foley, 2006a,b; Wang et al., 2002, 2008; Takači et al., 2009; van Reis and Saksena, 1997; Wallberg et al., 2003). However, only a few works have considered concentrationdependent rejections in the optimization procedure (Bowen and Mohammad, 1998; Kovács and Discacciati, 2008; Kovács et al., 2008). Assuming constant rejections might lead to inaccurate simulation and subsequent optimization results under conditions where the rejections of solutes are strongly vary depending on their feed concentrations and a considerably interdependence in their permeation occurs. 
We introduce a general method to obtain the optimal wash-water utilization strategy of diafiltration. We propose a rigorous dynamical model of the diafiltration process with concentration-dependent rejections of solutes. Based on the model, we define a problem of optimal process operation and rewrite it in a dynamic optimization formulation. Finally, we solve the problem and show that optimal process operation needs not to be any of the classical methods.

\section{Theory}

\subsection{Configuration of batch diafiltration}

The schematic representation of a batch membrane filtration system is shown in Fig. 1. In a batch operation, the retentate stream is recirculated to

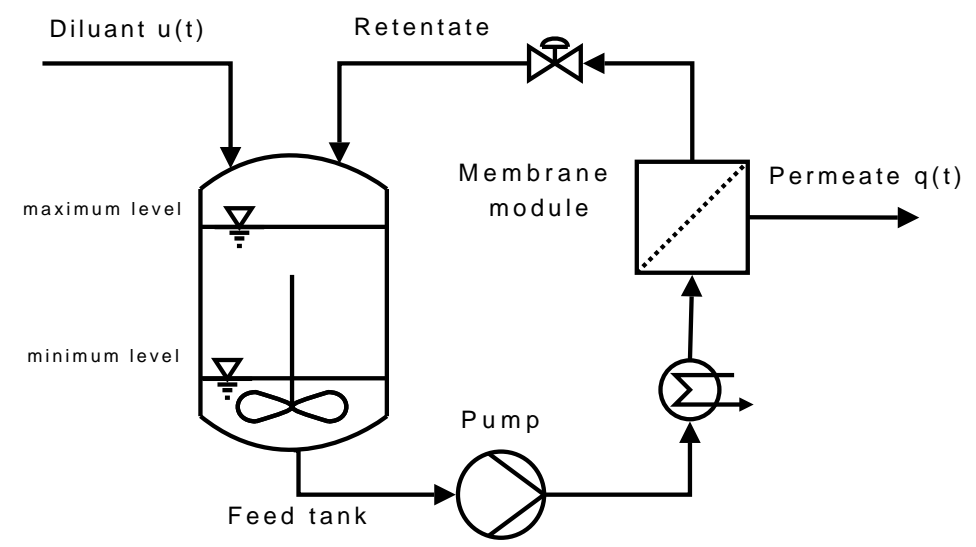

Figure 1: Schematic representation of diafiltration settings.

the feed tank, and the permeate stream $q(t)$ is collected separately. During the operation, fresh solute-free diluant stream $u(t)$ can be added into the feed tank. The proportionality factor $\alpha(t)$ is defined as the ratio of diluant flow $u(t)$ to permeate flow $q(t)$ :

$$
\alpha(t)=\frac{u(t)}{q(t)}
$$

The requirement for an effective separation is the utilization of a membrane which highly retains certain species (commonly referred to as macrosolutes) 
but permeable for other components (called as microsolutes). It is assumed that the rejections of both microsolute and macrosolute are affected by the extent to which the microsolute concentration is reduced and also to which the macrosolute is concentrated. Analogously, the permeate flux also depends on the actual feed concentration of both components.

\subsection{Mathematical modeling}

An essential stage in the development of the model is the formulation of appropriate mass balance equations. In this section we derive the governing

differential equations for diafiltration. The change in the feed volume during the operation is given as

$$
\frac{\mathrm{d} V_{f}}{\mathrm{~d} t}(t)=u(t)-q(t)
$$

In the following we assume that the diluant consists of no solutes. For modeling semi-batch processes we refer the reader to our previous study (Kovács et al., 2009a). Considering two solutes and a well-mixed feed tank, the mass balance for the solute concentrations yields

$$
\frac{\mathrm{d}}{\mathrm{d} t} V_{f}(t) c_{f, i}(t)=-q(t) c_{p, i}(t) \quad i=1,2
$$

where $c_{p, i}(t)$ denotes the permeate concentration of solute $i$ at time $t$. Equation (3) can be rewritten in the following way:

$$
\frac{\mathrm{d} V_{f}}{\mathrm{~d} t}(t) c_{f, i}(t)+V_{f}(t) \frac{\mathrm{d} c_{f, i}}{\mathrm{~d} t}(t)=-q(t) c_{p, i}(t) \quad i=1,2
$$

Using Eq. (2) and recalling that $c_{p, i}(t)=c_{f, i}(t)\left(1-\mathcal{R}_{i}(t)\right)$, where $\mathcal{R}_{i}(t)$ is the rejection of solute $i$ at time $t$, we obtain, for $i=1,2$,

$$
V_{f}(t) \frac{\mathrm{d} c_{f, i}}{\mathrm{~d} t}(t)=c_{f, i}(t)\left[q(t) \mathcal{R}_{i}(t)-u(t)\right] .
$$

Thus, we have the following initial-value problems:

$$
\left\{\begin{array}{l}
\frac{\mathrm{d} V_{f}}{\mathrm{~d} t}(t)=u(t)-q(t) \\
V_{f}(0)=V_{f}^{0}
\end{array}\right.
$$


and, for $i=1,2$,

$$
\left\{\begin{array}{l}
V_{f}(t) \frac{\mathrm{d} c_{f, i}}{\mathrm{~d} t}(t)=c_{f, i}(t)\left[q(t) \mathcal{R}_{i}(t)-u(t)\right] \\
c_{f, i}(0)=c_{f, i}^{0}
\end{array}\right.
$$

which describe the evolution in time of the volume in the feed tank $V_{f}$ and of the feed concentration $c_{f, i} . V_{f}^{0}$ and $c_{f, i}^{0}$ denote respectively the initial feed volume and the initial feed concentration of the solute $i$. An overview of the analytical solutions of Eqs. 4 and 5 for the special cases of C, CVD, and VVD assuming constant rejection values are reported in (Kovács et al., 2009b). In many applications, the rejections are concentration-(inter)dependent quantities. In such cases, no closed form solution exists, thus, numerical techniques are required to solve the model equations.

\subsection{Membrane response}

The separation behavior of the membrane can be characterized in terms of permeate flux and solute rejections. One of the advantages of the presented modeling approach is that the design equations describing the overall mass balance of the plant configuration are handled separately from the estimation methods describing the mass transfer through the membrane. The estimation of the flow $q(t)$ and of the rejection $\mathcal{R}_{i}(t)$ presented in Eqs. 4 and 5 can be carried out separately using the most convenient approach for the problem at hand. Either mechanism-driven or data-driven models can be employed. Mechanism-driven models are based on a physical understanding of the transport phenomenon. In contrast with that, data-driven models make a direct use of the experimental data obtained from filtration tests with the process liquor.

For further mathematical analysis, we use the filtration data from our earlier work (Kovács et al., 2009a). We consider relations for $q$ and $\mathcal{R}$ obtained from nanofiltration experiments with the membrane Desal-DK5 separating a binary aqueous solution at constant temperature and pressure. The process liqueur was a test system consisting of sucrose (solute 1) and sodium chloride (solute 2). The nanofiltration apparatus, the sample analysis, and possible mechanism-driven and data-driven models to quantify membrane response have been described in detail in (Kovács et al., 2009a). The empirical 
Table 1: Experimentally obtained coefficient values for $\mathcal{R}$ and $q$.

\begin{tabular}{cccc} 
& $s$ & $w$ & $z$ \\
\hline 1 & $68.125010^{-9}$ & $7.840710^{-6}$ & $-0.076910^{-6}$ \\
2 & $-56.451210^{-6}$ & $-4.050710^{-3}$ & $-0.003510^{-3}$ \\
3 & $32.555310^{-3}$ & 1.0585 & $0.034910^{-3}$ \\
4 & $-4.352910^{-9}$ & $1.231810^{-9}$ & 0.9961 \\
5 & $3.321610^{-6}$ & $-9.766010^{-6}$ & \\
6 & $-2.714110^{-3}$ & $-1.167710^{-3}$ &
\end{tabular}

relations for $q$ and $\mathcal{R}_{i}$ as functions of feed composition are as follows:

$$
\begin{aligned}
q & =S_{1}\left(c_{2}\right) \mathrm{e}^{S_{2}\left(c_{2}\right) c_{1}} \\
\mathcal{R}_{1} & =\left(z_{1} c_{2}+z_{2}\right) c_{1}+\left(z_{3} c_{2}+z_{4}\right) \\
\mathcal{R}_{2} & =W_{1}\left(c_{2}\right) \mathrm{e}^{W_{2}\left(c_{2}\right) c_{1}}
\end{aligned}
$$

where $S_{1}, S_{2}, W_{1}, W_{2}$ are second order polynomials in $c_{2}$

$$
\begin{aligned}
S_{1}\left(c_{2}\right) & =s_{1} c_{2}^{2}+s_{2} c_{2}+s_{3} \\
S_{2}\left(c_{2}\right) & =s_{4} c_{2}^{2}+s_{5} c_{2}+s_{6} \\
W_{1}\left(c_{2}\right) & =w_{1} c_{2}^{2}+w_{2} c_{2}+w_{3} \\
W_{2}\left(c_{2}\right) & =w_{4} c_{2}^{2}+w_{5} c_{2}+w_{6}
\end{aligned}
$$

and $s_{1-6}, z_{1-4}, w_{1-6}$ are coefficients that were determined from laboratory experiments with the process solution (see Table 1).

\subsection{Dynamic-volume diafiltration}

The widely applied diafiltration techniques differ in controlling the quantity and the duration of the diluant stream introduced in the feed tank. During an operational mode, the diluant flow can be set to zero, or alternatively, it can be equal or proportional to the permeate flow rate. The $\alpha$ formulation was used to illustrate the control strategies of some common diafiltration processes by Foley (Foley, 2006b). We adopt this schematic representation, and in Fig. 2 we show the $\alpha(t)$ versus time profiles for the three conventional diafiltration processes. In TD, PVVD, and IFD, arbitrarily constructed schemes for water usage are applied. For example, TD process is characterized with a sequence $\alpha(t)=\{0,1,0\}$ and there are two unknown 
Figure 2: Typical control profiles for traditional diafiltration (TD), preconcentration combined variable-dilution mode (PVVD), and intermittent feed diafiltration (IFD) processes. In IFD, dilution is achieved by rapid mixing of the process liquor with wash-water.

switching times at the ends of the first and the second time interval. Similarly, PVVD process has two phases with constant $\alpha$ levels $\alpha(t)=\left\{0, \alpha_{1}\right\}$ with variables $0<\alpha_{1}<1$ and a switching time after which the the ratio of diluant flow to permeate flow is held constant.

It should be pointed out, that the best time-varying profile of the diluant addition needs not necessarily be one of the pre-defined profiles of TD, PVVD or IFD. The optimal control trajectory of the diluant flow $u(t)$ (or equivalently $\alpha(t)$ ) can be determined by formulating an optimization problem subject to process model described by differential equations. The diafiltration process, that is designed by the evaluation of the optimal time-varying profile of the diluant flow, is referred to as dynamic-volume diafiltration (DVD) in the rest of this paper. In this context, all conventional diafiltration processes are specific cases of DVD. 


\subsection{Definition of optimal operation}

Optimality is defined as the minimization of the objective function without violating given constraints. In the following, we use the processing conditions and the specifications of our laboratory system for the optimization task. However, the concept can find a general interest, and industrial problems can be handled in an analogous way. This technique can be useful to find the optimal operational parameters of an existing membrane plant with a defined membrane area.

\subsubsection{Objective function}

We assume two case problems with different cost functions:

Case A. minimization of the final concentration of microsolute concentration at a fixed final time of operation. This can equivalently be described as

$$
\min _{u(t)} J_{A}=c_{2}\left(t_{f}\right)
$$

Case $B$. the most economical process described as minimization of a mixed objective involving operational cost of the pump, the cost of the loss of the macrosolute component, and the cost of the utilized dilution water. This yields

$$
\begin{aligned}
\min _{u(t)} J_{B} & =k_{1} t_{f}+k_{2} \int_{0}^{t_{f}} c_{p, 1}(t) q(t) \mathrm{d} t+k_{3} \int_{0}^{t_{f}} u(t) \mathrm{d} t \\
& =\int_{0}^{t_{f}} k_{1}+k_{2} c_{1}\left(1-\mathcal{R}_{1}(t)\right) q(t)+k_{3} u(t) \mathrm{d} t
\end{aligned}
$$

The first term of Eq.14 expresses the operational cost of the pump, where the constant $k_{1}$ is a product of the power consumption of the pump and the electricity price, which gives $k_{1}=7.5 \mathrm{kWh} \times 0.07 € / \mathrm{kWh}=0.525 € / \mathrm{h}$ considering our laboratory test conditions. The second term represents the cost of the mass loss of the valuable component during the entire process. This is calculated by integrating the permeating mass of valuable components through the membrane over the process time. In our illustrative example, the price of commercial table sugar was used to determine the constant $k_{2}$ resulting in $k_{2}=0.3423 € / \mathrm{mol}$. Finally, the third term is introduced to account for the cost of the utilized diluant by integrating the permeate flux over the process time. Here, the constant $k_{3}=10 € / \mathrm{m}^{3}$ is taken as unit price of the utilized dilution water. 
In both cases, the optimized variable is either diluant flow $u(t)$ or the proportionality factor $\alpha(t)$ tied together with relation (1). The conventional diafiltration techniques are described best using $\alpha$ formulation as shown in Fig. 2.

Traditional diafiltration process are often optimized by minimizing the total process time. This represents an optimization problem that is a specific case of Case B. If the cost of the mass losses of the valuable component and the cost of the diluant are negligible, then the values used for $\mathrm{k}_{2}$ and $\mathrm{k}_{3}$ can be set to zero. Thus, the second and the third term in Eq.14 vanish, and Case $\mathrm{B}$ reduces to a time minimization problem.

\subsubsection{Constraints}

The initial macro- and microsolute concentrations are $c_{f, 1}^{0}=150 \mathrm{~mol} / \mathrm{m}^{3}$ and $c_{f, 2}^{0}=300 \mathrm{~mol} / \mathrm{m}^{3}$, respectively. The initial volume of $V^{0}=0.03 \mathrm{~m}^{3}$ is to be reduced to $0.01 \mathrm{~m}^{3}$ at the final time. A lower and an upper volume threshold level is defined based on the size of the feed tank, which can not be exceeded during the operation. A safe operation is ensured when the volume in the feed tank is within 0.01 and $0.035 \mathrm{~m}^{3}$. The dilution water is supplied with an external pump with maximum flow-rate $1.0 \mathrm{~m}^{3} / \mathrm{h}$.

The constraints are thus given as follows

$$
\begin{aligned}
V\left(t_{f}\right) & =0.01 \\
V(t) & \in[0.01,0.035] \\
u(t) & \in[0,1]
\end{aligned}
$$

In Case $A$, we have defined the operation time as $t_{f}=6 \mathrm{~h}$, and there is no constraint given on the final microsolute concentration. In contrast with that, Case $B$ is an open final time problem. Here, the final microsolute concentration $c_{f, 2}\left(t_{f}\right)$ is to be reduced to a limit value of $50 \mathrm{~mol} / \mathrm{m}^{3}$.

\subsection{Dynamic optimization formulation}

As we can see from the previous sections, the studied optimal diafiltration operation can be described as a dynamic optimization problem. If we define states $x_{1}=c_{1}, x_{2}=c_{2}$, and $x_{3}=V$ and optimized variable $u(t)$, the formulation for the case $\mathrm{A}$ is as follows

$$
\min _{u(t)} J_{A}=x_{2}\left(t_{f}\right)
$$


subject to differential equations:

$$
\begin{array}{llrl}
\dot{x}_{1} & =\frac{x_{1}}{x_{3}}\left[q\left(x_{1}, x_{2}\right) \mathcal{R}_{1}\left(x_{1}, x_{2}\right)-u\right], & x_{1}(0) & =150 \\
\dot{x}_{2} & =\frac{x_{2}}{x_{3}}\left[q\left(x_{1}, x_{2}\right) \mathcal{R}_{2}\left(x_{1}, x_{2}\right)-u\right], & x_{2}(0) & =300 \\
\dot{x}_{3} & =u-q\left(x_{1}, x_{2}\right), & x_{3}(0) & =0.03
\end{array}
$$

state path constraints:

$$
\begin{aligned}
& x_{3}(t) \geq 0.01 \\
& x_{3}(t) \leq 0.035
\end{aligned}
$$

final time constraints:

$$
x_{3}\left(t_{f}\right)=0.01
$$

and simple bound constraints on optimized variable

$$
u(t) \in[0,1]
$$

The formulation for the case B follows analogously using state $x_{4}$ to transform original integral cost function to Meyer form:

$$
\min _{u(t)} J_{B}=x_{4}\left(t_{f}\right)
$$

where:

$$
\dot{x}_{4}=k_{1}+k_{2} x_{1}\left(1-\mathcal{R}_{1}\left(x_{1}, x_{2}\right)\right) q\left(x_{1}, x_{2}\right)+k_{3} u, \quad x_{4}(0)=0
$$

\section{Simulation Results and Discussion}

There are various methods and toolboxes suitable for solving dynamic optimization problems. Modern numerical methods can be divided according to a degree of approximation of the original continuous-time to a problem solvable by a nonlinear programming (NLP) tools. Two major groups are represented by orthogonal collocation (OC) methods where both states and control are approximated as piece-wise polynomials on finite time intervals (Cuthrell and Biegler, 1987; Logsdon and Biegler, 1989; Avraam et al., 1998) and by control vector parametrization (CVP) methods where only control is approximated and states are solved in integration loop (Goh and Teo, 
1988; Balsa-Canto et al., 2001; Vassiliadis et al., 1994; Fikar and Latifi, 2002; Hirmajer et al., 2009).

Both groups have advantages and drawbacks. In general, OC produces a larger NLP formulation and is of infeasible type, where solution is obtained only if optimum is found. On the other side, CVP spends a large fraction of time in solution of differential equations even for a combination of optimized parameters that is far from the optimum. Moreover, state path constraints are more difficult to take into account compared to OC methods.

We have applied freely available package Dynopt (Čižniar et al., 2005) implemented in Matlab. It is based on OC methods as our problem includes state path constraints and contains only a few differential equations.

\subsection{Case $A$}

Simulation results of DVD process are shown in Fig. 3. Minimum concentration of $x_{2}(6)=23.38 \mathrm{~mol} \mathrm{~m}^{-3}$ is obtained with 3 piece-wise linear profiles. The optimal control profile is at zero for the first part of trajectory and then slowly increases for the rest. This is translated to the trajectory of $\alpha(t)$ that is zero at the beginning and approximately equal to one after the switch. Inspection of the volume shows that the first part of the trajectory basically decreases the volume until it is on the lower constraint and keeps it approximately constant until end of the batch. Thus, the optimal control strategy for this problem represents a TD process with two parts: pre-concentration followed by approximately constant-volume step until end of the batch.

We have reformulated the problem using the proportionality factor $\alpha$ as the optimized variable instead of the permeate flow $u$ so that the optimal control trajectory can guarantee the constant volume step in a more natural way. Slightly better minimum concentration of $x_{2}(6)=23.13 \mathrm{~mol} \mathrm{~m}^{-3}$ was obtained with 2 piece-wise constant profiles of $\alpha$.

Optimization with different fixed final times between $t_{f}=[4,13] \mathrm{h}$ confirms the structure of the optimal control trajectory represented by TD process as described above. Based on this it is relatively easy to select the final time of operation if a certain decrease of $x_{2}$ at the end of the batch is desired.

\subsection{Case $B$}

Minimum value of the cost function obtained is $J_{B}=2.65$ with 3 piecewise constant control profiles and final time $t_{f}=4.50 \mathrm{~h}$. Results indicate that the optimal control operation is the same as in Case A and it represents a two-step TD process. 

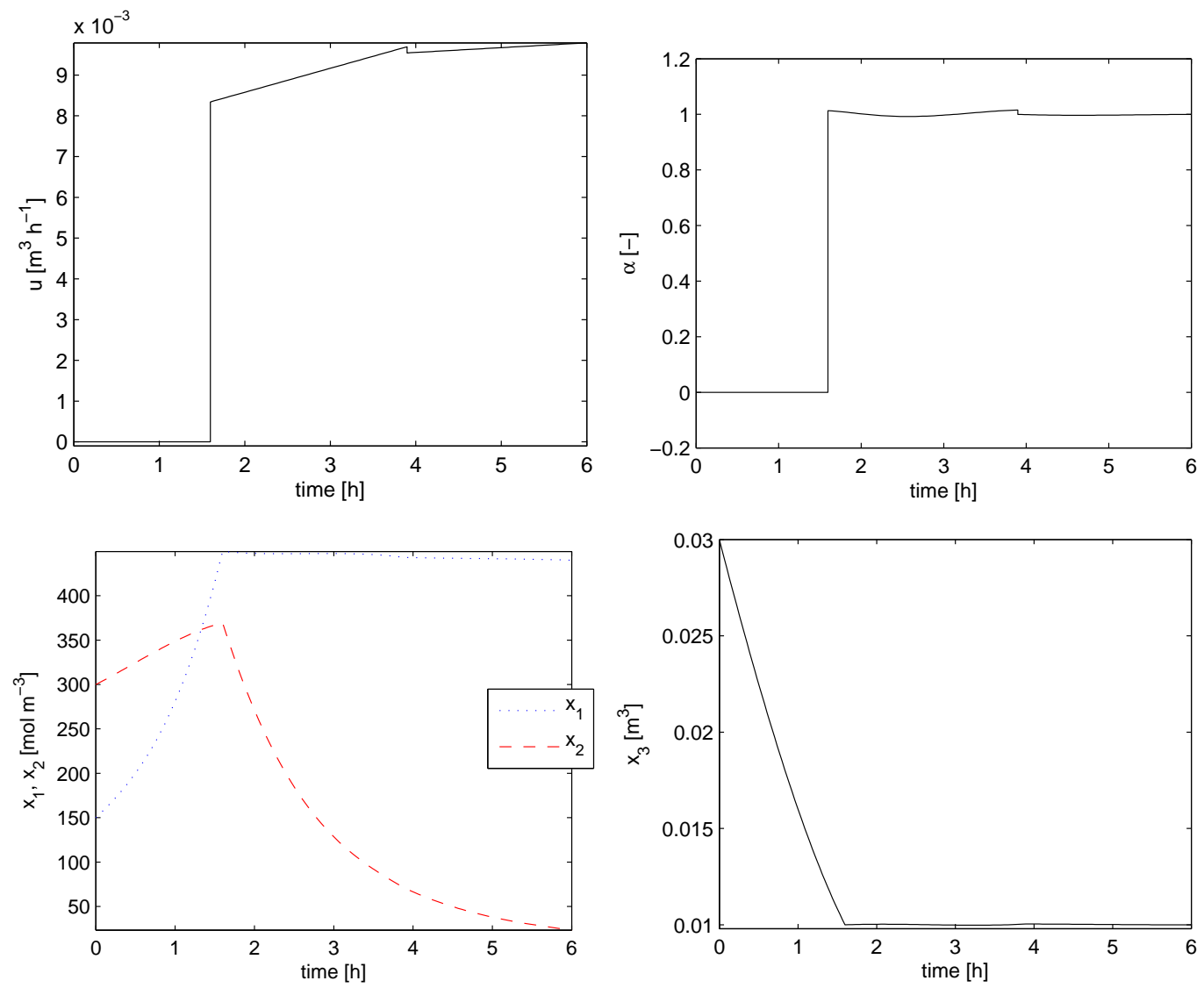

Figure 3: Problem A: optimal control (top left), optimal $\alpha$ (top right), concentrations (bottom left), and volume (bottom right) as functions of time

\subsection{Modified problem}

Conventional diafiltration processes are created involving C, CVD and VVD. Note, that many different sequences of consecutive operational steps can be thought as possible diafiltration concepts. Traditionally, these individual processes were analyzed and then compared in order to determine the optimal settings. The proposed optimization procedure eliminates the need for that since it readily provides the overall optimal control.

In the next scenario, we have assumed a membrane with lower water permeability. For simplicity, we have modeled this with an increase of the parameter $s_{6}$, which is now assumed three times larger compared to the nominal case. As the membrane is less permeable, we have assumed final 
time given as $t_{f}=50 \mathrm{~h}$ if the case $\mathrm{A}$ was considered. The rejection performance is left unchanged. This scenario is fictitious, however, realistic from the point of view of an experimentalist. In practice, a great diversity of membrane/solute/solvent systems occurs which allows us the creation of such an arbitrary scenario for further mathematical analysis. Our intention is to demonstrate that the optimal process operation needs not to be any of the conventional techniques. In fact, the computed optimal control is very sensitive to changes in the membrane performance.

Minimum concentration of $x_{2}(50)=9.30 \mathrm{~mol} \mathrm{~m}^{-3}$ is obtained with 2 piece-wise parabolic profiles and results are shown in Fig. 4. The optimal control profile is between $4-5 \cdot 10^{-3}$ for almost all time and zero in the last $3.5 \mathrm{~h}$. The trajectory of $\alpha$ variable no longer represents TD process. It consists of two phases. In the first one $\alpha$ increases exponentially from 0.71 to 1. The switch occurs when concentration $x_{2}$ no longer decreases and obtains its minimum (about $6.30 \mathrm{~mol} \mathrm{~m}^{-3}$ ). At this instant, $\alpha$ is set to zero. The volume is reduced until it satisfies the final constraint.

The same type of control profile has been obtained in the case B. An important difference between case $\mathrm{A}$ and case $\mathrm{B}$ is that the cost function of case B (Eq. 14) includes a macrosolute-dependent term. In our specific solute/membrane system, however, the impact of the macrosolute loss on the total costs is negligible due to an almost complete macrosolute rejection. Although the optimal trajectory depends on many factors in a complex manner, it can be assumed that especially in situations, where a pronounced $\mathcal{R}_{2}$ decline with increasing $c_{f, 2}$ occurs, different optimal controls may be obtained for case A and case B type problems.

In this study, we have employed empirical relations (Eqs. 6-12) to represent the membrane response. Note, that the provided optimization technique is not restricted to the analysis of this type of data-driven membrane model. The mechanism-driven NF models, such as irreversible thermodynamics models (Ahmad et al., 2005; Kovács et al., 2009c) or electrokinetic space-charge models (Bowen and Mohammad, 1998; Geraldes and Alves, 2008), consist of numerous working equations and iterative solution procedures. Dynamic optimization involving such complex equation-systems would be a difficult task. This problem can be avoided as follows. Mechanism-driven models can be first used to compute a set of $\mathcal{R}$ and $q$ data for the feed composition-region of interest. Then, a curve fitting procedure can be applied to derive simple relations in a similar form of Eqs. (6)-(12). This alternative method allows 

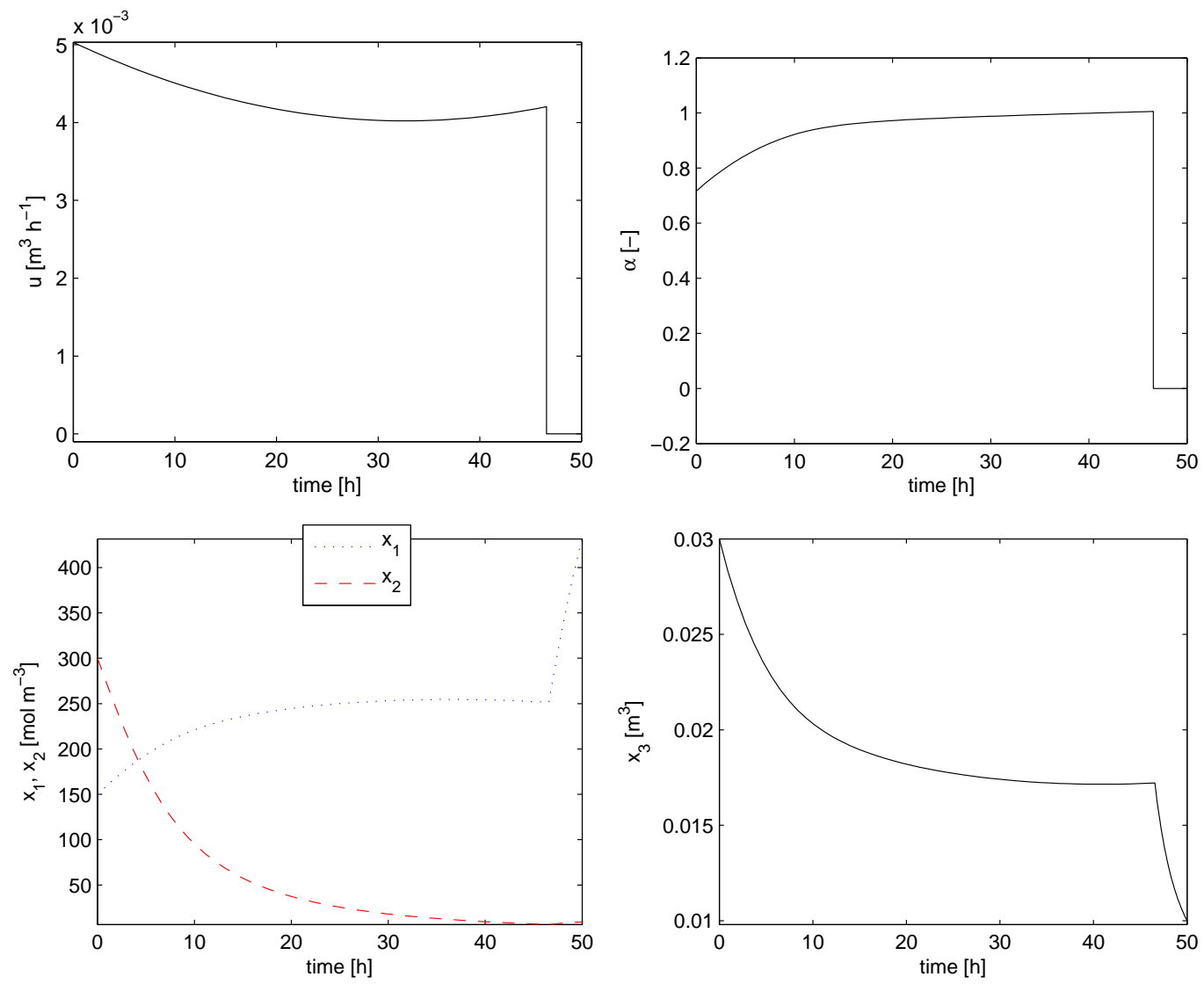

Figure 4: Modified problem A (changed $s_{6}$ ): optimal control (top left), optimal $\alpha$ (top right), concentrations (bottom left), and volume (bottom right) as functions of time

the dynamic optimization formulation of mechanism-driven models.

The here presented methodology for designing a DVD process is general in the sense that it can be readily adopted for different solute/membrane systems without the need of major changes in the provided procedure. However, the output of the optimization is unique for each application. The strategy of diluant utilization depends primary on

1. the response of the particular membrane to the specific solution that is expressed in terms of rejection $\mathcal{R}_{i}$ and permeate flow $q$,

2. the terms involved in the objective function (i.e. the definition of the separation goal),

3. the numerical values of the cost factors $k_{1}, k_{2}$, and $k_{3}$ in the objective 
function,

4. the constraints involved and their numerical values that need to be satisfied.

Any changes in these above listed specifications may modify the output of the optimization and lead to a different optimal control strategy. The presented modeling approach is flexible in its application to scenarios with modified settings and it permits rapid evaluation of the overall optimal control trajectory.

\title{
4. Conclusion
}

We have presented a detailed mathematical model that can be used for simulation, optimization, and control of diafiltration processes. It unifies the existing models for classical diafiltration concepts. The model was used for determination of optimal operation of a specific separation design problem. Methods of dynamic optimization were employed to obtain optimal solutions. We have shown that conventional diafiltration techniques can but need not be optimal. The presented methodology is particularly applicable for decision makers to evaluate the optimal water utilization strategy for the given separation design problem.

\section{Appendix A. List of Symbols}

\author{
c concentration $\left(\mathrm{mol} \mathrm{m} \mathrm{m}^{-3}\right)$ \\ $J \quad$ objective function \\ $k$ coefficients of cost function as defined in the text \\ $q$ permeate flow-rate $\left(\mathrm{m}^{3} \mathrm{~h}^{-1}\right)$ \\ $\mathcal{R}$ rejection \\ $t \quad$ operation time $(\mathrm{h})$ \\ $u$ diluant flow-rate $\left(\mathrm{m}^{3} \mathrm{~h}^{-1}\right)$ \\ $x \quad$ state variables $\left(\mathrm{mol} \mathrm{m}^{-3}\right)$ \\ $V \quad$ volume $\left(\mathrm{m}^{3}\right)$ \\ Greek symbols \\ $\alpha \quad$ proportionality factor of diluant flow to permeate flow \\ Subscripts \\ $A$ case problem $\mathrm{A}$ as described in the text \\ $B \quad$ case problem $\mathrm{B}$ as described in the text
}




\section{Appendix B. Acknowledgment}

This research is a cooperative effort. The first author acknowledges the support of the Slovak Research and Development Agency under the contract No. VV-0029-07. The second author would like to thank the Hessen State Ministry of Higher Education, Research and the Arts for the financial support within the Hessen initiative for scientific and economic excellence (LOEWEProgram).

\section{References}

M. Jaffrin, J. Charrier, Optimization of ultrafiltration and diafiltration processes for albumin production, Journal of Membrane Science 97 (1994) 71 $-81$.

M. Tekić, Z. Zavargó, D. Krstić, M. Djurić, Mathematical model of variable volume diafiltration, Hung. J. Indus. Chem. 30 (2002) 211-214.

D. Krstić, M. Tekić, Z. Zavargó, M. Djurić, G. Ćirić, Saving water in a volume-decreasing diafiltration process, Desalination 165 (2004) 283-288.

G. Foley, Water usage in variable volume diafiltration: comparison with ultrafiltration and constant volume diafiltration, Desalination 196 (2006a) 160-163. 
G. Foley, Ultrafiltration with variable volume diafiltration: a novel approach to water saving in diafiltration processes, Desalination 199 (1-3) (2006b) $220-221$.

L. Wang, G. Yang, W. Xing, N. Xu, Mathematic model of the yield for diafiltration processes, Sep. Purif. Technol. 59 (2008) 206-213.

A. Takači, T. Žikić-Došenović, Z. Zavargó, Mathematic model of variable volume diafiltration with time dependent water adding, Engineering Computations 26 (2009) 857-867.

X.-L. Wang, C. Zhang, P. Ouyang, The possibility of separating saccharides from a $\mathrm{NaCl}$ solution by using nanofiltration in diafiltration mode, Journal of Membrane Science 204 (1-2) (2002) 271 - 281.

R. van Reis, S. Saksena, Optimization diagram for membrane separations, Journal of Membrane Science 129 (1) (1997) 19 - 29.

O. Wallberg, A. Joensson, R. Wimmerstedt, Fractionation and concentration of kraft black liquor lignin with ultrafiltration, Desalination 154 (2003) 187-199.

W.R. Bowen, A.W Mohammad, Diafiltration by Nanofiltration: Prediction and Optimization, AIChE Journal 44 (8) (1998) 1799-1812.

Z. Kovács, M. Discacciati, Modelling of multi-step microfiltration process for solvent exchange, Hungarian Journal of Industrial Chemistry 36 (2008) 65-69.

Z. Kovács, M. Discacciati, W. Samhaber, Numerical simulation and optimization of multi-step batch membrane processes, J. Membr. Sci. 324 (2008) 50-58.

Z. Kovács, M. Discacciati, W. Samhaber, Modeling of batch and semi-batch membrane filtration processes, J. Membr. Sci. 327 (2009a) 164-173.

Z. Kovács, M. Fikar, P. Czermak, Mathematical modeling of diafiltration, Hungarian Journal of Industrial Chemistry (2009b) Accepted.

J. E. Cuthrell, L. T. Biegler, On the optimization of differential-algebraic process systems, AIChE J. 33 (8) (1987) 1257-1270. 
J. S. Logsdon, L. T. Biegler, Accurate Solution of Differential-Algebraic Optimization Problem, Ind. Eng. Chem. Res. 28 (1989) 1628-1639.

M. P. Avraam, N. Shah, C. C. Pantelides, Modelling and Optimisation of General Hybrid Systems in the Continuous Time Domain, Computers chem. Engng. 22 (Suppl.) (1998) S221-S228.

C. J. Goh, K. L. Teo, Control parameterization: a unified approach to optimal control problems with general constraints, Automatica 24 (1) (1988) $3-18$.

E. Balsa-Canto, J. R. Banga, A. A. Alonso, V. S. Vassiliadis, Dynamic optimization of chemical and biochemical processes using restricted secondorder information, Computers chem. Engng. 25 (4-6) (2001) 539-546.

V. S. Vassiliadis, R. W. H. Sargent, C. C. Pantelides, Solution of a Class of Multistage Dynamic Optimization Problems. 1. Problems without Path Constraints, 2. Problems with Path Constraints, Ind. Eng. Chem. Res. 33 (1994) 2111-2122, 2123-2133.

M. Fikar, M. A. Latifi, User's Guide for FORTRAN Dynamic Optimisation Code DYNO, Tech. Rep. mf0201, LSGC CNRS, Nancy, France; STU Bratislava, Slovak Republic, 2002.

T. Hirmajer, E. Balsa-Canto, J. R. Banga, DOTcvpSB, a software toolbox for dynamic optimization in systems biology, BMC Bioinformatics 10 (1) (2009) 199.

M. Čižniar, M. Fikar, M. A. Latifi, MATLAB Dynamic Optimisation Code DYNOPT. User's Guide, Technical Report, KIRP FCHPT STU Bratislava, Slovak Republic, 2005.

A. Ahmad, M. Chong, S. Bhatia, Mathematical modeling and simulation of the multiple solutes system for nanofiltration process, J. Membr. Sci. 253 (2005) 103-115.

Z. Kovács, M. Discacciati, W. Samhaber, Modeling of amino acid nanofiltration by irreversible thermodynamics, J. Membr. Sci. 332 (2009c) 38-49.

V. Geraldes, A. Alves, Computer program for simulation of mass transport in nanofiltration membranes, J. Membr. Sci. 321 (2008) 172-182. 